\title{
Is mainstreaming always the answer? The social and economic development of service user communities
}

\author{
James M. Mandiberg, ${ }^{1}$ Richard Warner ${ }^{2}$
}

The Psychiatrist (2013), 37, 153-155, doi: 10.1192/pb.bp.112.040659

${ }^{1}$ Columbia University, New York, USA; ${ }^{2}$ University of Colorado, USA

Correspondence to James M. Mandiberg (email: jmm2151@columbia.edu)

First received 29 Jun 2012, final revision 26 Nov 2012, accepted 10 Jan 2013

\begin{abstract}
Summary The community placement of people with serious mental illness has focused on assimilation. The broad community, however, seems largely unwilling to support and include them. The result is programmes to help people maintain their community lives mainly through the support of formal mental health services. The social and economic development of 'identity communities' of people with serious mental illness is an alternative model to assimilation. An identity community consists of people with shared interests, beliefs, experiences or needs which affect the identity of the participants and the cohesiveness of the group. Such communities can be economically sustainable and offer members more ways of having meaningful lives. Examples of this recovery-friendly approach are provided as a viable way of enhancing community support without additional expense.
\end{abstract}

Declaration of interest J.M.M. has received NIH origin grant funding for research on training peer specialist mental health providers.
In the initial stages of deinstitutionalisation in the USA, thousands of people were dumped from hospitals into lowincome and underresourced communities. The situation was so dire that, in 1977, the US National Institute of Mental Health created the Community Support Program, a model supporting people with serious mental illness in the community. The Program proposed that the broad community should provide the supports that people need, with the assistance of formal mental health programmes that help people gain basic life skills and provide ongoing treatment. The goal was for people with serious mental illness to live, work and socialise in the 'normal' community. This has been called mainstreaming, normalisation, integration and, most recently, inclusion. The UK developed a similar social policy of community or domiciled care. In the UK, implementing a community $v$. an institutional approach included shifting responsibility to local authorities, combining health and social care, reducing costs to the government, and innovating private sector organisations and care models.

In both cases, the broad community was not asked whether they were willing to provide the supports people with a history of serious mental illness would need to live, work and socialise among them. Similarly, people with a history of serious mental illness were not asked whether assimilation into the broad community was what they desired.

\section{Current community support models}

Experience with the community support approach reveals that most in the broad community are not willing to provide the level of support that many of those with serious mental illness require. The experience also demonstrates that although some people with serious mental illness would like to live, work and socialise predominately in the broad community, others prefer primarily to be among those who share their experiences of mental illness, and still others prefer to move back-and-forth between the broad community and the community of their peers.

Although the broad community would not provide it, some form of support is necessary for people to maintain their community lives. Consequently, new professionally delivered mental health service models emerged to fill this need. These include such well-known models as assertive community treatment (ACT), supported employment, supported housing and supported education. One feature of these models is that they are not time limited; rather, they provide support for as long as the service user needs it. Thus, for many people with serious mental illness, their living, working and socialising in the community is maintained principally through funded, professionally provided supports.

Receiving ongoing support from publicly funded programmes is an expensive and often unsuccessful way to help people with serious mental illness lead rewarding lives in the community. People with psychiatric disabilities who are living and working in the broad community are often described as being 'in but not of' the community and the workplace. That is, they may be physically present, but they are not fully included. For inclusion to work, both sides must agree to it. Stigma, both external and internalised, is a major barrier to inclusion. Large-scale national and international anti-stigma campaigns have not proven particularly successful and, although there has been some 
progress in finding ways to influence people's stigmatised beliefs at a local level, ${ }^{1,2}$ it is unclear how well this translates into action and willingness to include. Meanwhile, the sense of otherness and incapacity that result from internalised stigma may best be overcome by productive and social activity within one's own community. ${ }^{3}$

Using funded services to maintain people's community presence is problematic. Permanent support models are dependency building. Dependency is not necessarily bad in and of itself, since everyone depends on others in their daily lives. Building dependency on funded services is risky, however, since funding support is limited and variable. What is needed is an economically viable approach to meeting the support needs of those with psychiatric disabilities.

\section{An alternative approach}

Many have advocated private-sector and market-based solutions to social welfare problems. In the UK, this has included expansion of the non-profit sector; development of social enterprise-based services; and innovative funding strategies such as social impact bonds. These are laudable but are principally focused on cost-cutting, cost-shifting and efficiency, all within the same paradigm. Something more fundamental is needed than a different way of funding the same type of services.

Finding ways to help people live successfully in the community requires us to change focus from mental illness and disability and to refocus on the community. Is there a community to which people with psychiatric conditions belong, not as a result of wishful thinking and as yet unsuccessful anti-stigma campaigns, but one in which they have unconditional acceptance? Perhaps it is most fruitful to begin to answer this by looking at how other socially excluded populations achieve community and inclusion.

\section{Enclave community}

One approach to this looks at how immigrants adjust to a new country. ${ }^{4}$ Typically, many immigrants form enclave communities rather than living, working and socialising in the broader community. Enclave communities give immigrants opportunities to build businesses and business aptitude, gain leadership skills and experiences, and create civil society activities and institutions. The broad community provides few similar opportunities for immigrants because of stigma, prejudice and unequal access. As an alternative to their inevitably contested membership in the broad community, some immigrants opt for unconditional membership in their own enclave communities. Once immigrant communities have leaders, businesses, and civil society and political activities, the broad community is compelled to include them. That is, by building enclave communities immigrants do not wait to be included. Rather, they build successful community lives that lead to natural inclusion, not imposed inclusion as a result of government policy.

From these enclave communities, individuals then decide whether to assimilate into the broad community, remain in the enclave community, or move back-and-forth between the broad and enclave communities. Research demonstrates that social minorities in general utilise these same three approaches in their relationships to the majority. ${ }^{5}$ Currently in mental health services, only one of these ways of leading a community life is regarded as successful - assimilation. If these same three options were available to people with mental health conditions, it could result in more people leading successful lives in the community.

\section{Identity community}

Community, including enclave community, need not be defined solely by location. 'Identity communities' are formed based on interests, beliefs, experiences and needs which influence the identity of group members and the cohesiveness of the group. ${ }^{6}$ Examples include the deaf community and communities of practice such as psychiatric associations. People with serious mental health conditions also constitute an identity community. When the community potential of that identity community is recognised, it becomes possible to support it using a community development approach rather than a service-funded approach. Once created, communities can be largely socially and economically self-sustaining. Many community members maintain their lives in the community from the naturally occurring supports that are created by the businesses, institutions and social relations of the community. If a fully developed identity community of people with mental health conditions existed, it could provide a base-level of supports that funded programmes currently provide, but in an economically sustainable way. ${ }^{7}$

\section{What would a mental health identity community look like?}

\section{The California project}

As yet, there is no example of a fully developed identity community for people with mental health conditions, but there have been some demonstration projects that point to its viability. The Clustered Apartment Project in Santa Clara County, California, USA, clustered the housing of mental health service users to build mutual support among them. People lived within several minutes' walking distance of each other and a community gathering space was located in the geographic centre of the clustered housing. Community organisers (non-clinician professionals trained in developing communities) were hired instead of clinicians to help community members identify and meet their own needs. ${ }^{8}$ In one example, the community members replaced professionally delivered crisis and respite services by providing similar services to themselves. ${ }^{9}$ This approach is currently being used by one of the authors (R.W.) in a community living programme in Boulder, Colorado.

\section{Introducing business and civil society structures into identity communities}

The Clustered Apartment Project is not a fully articulated community because it lacks community business and civil society infrastructure. Several projects and mental health reform efforts have explored how to develop different aspects of business and civil society creation within mental health identity communities. ${ }^{10}$ We have previously reported on the development of an economic assessment model that analysed the assets of people with serious mental illness and where those assets were spent. Based on that analysis, a 
mental health pharmacy was established. The pharmacy offers better services and education for service users, provides jobs for service users as pharmacy technicians, and makes a profit which is used to support the development of other identity community infrastructure. Several projects have also explored how to incubate small businesses owned and operated by people with psychiatric disabilities. These projects found that people with psychiatric disabilities can successfully run small and micro-businesses, which provide self-employment and create a business infrastructure for the identity community.

The business incubation projects also found that one of the biggest obstacles to creating and growing small businesses owned by people with psychiatric disabilities is gaining access to capital. Entrepreneurs with a history of mental health service use often have poor credit histories, making conventional business loans impossible. Further, microcredit only capitalises the smallest businesses. As a solution, a mental health user-run organisation in New York State is creating a credit union, a form of community banking, for all residents of New York who have a history of mental health service use. The small deposits of individuals will create a leverageable corpus from which business loans can be extended to entrepreneurs from among the credit union's members. Just as the mental health pharmacy builds on the assets of the identity community, the credit union does the same. Both initiatives help to demonstrate how a community development approach is economically sustainable.

\section{The clubhouse and social cooperative models}

There are other examples of how a fully developed identity community might function. The psychosocial clubhouse model, developed at Fountain House in New York City in the late 1940s, is based on mutual support. There are now well over 300 such clubhouses in over 30 countries, including 8 in the UK (www.iccd.org/about.html). Mental health social cooperative businesses that exist in Italy and other European and non-European countries provide a different model for identity community business development. The social cooperatives in Trieste, Italy, are an important element of the local economy and, along with many other businesses, hold all of the street and public building cleaning contracts for the city. ${ }^{11}$ The number of social firms in the UK has increased from just 6 before 1997 to over 150 currently, most of them self-sustaining (www.socialfirmsuk.co.uk). Community development of this type demands new professional roles. The creation of successful social firms requires business management skills, capital and the ability to locate niche markets - elements beyond the resources of mental health service providers. A unique mental health community in northern Japan, Bethel House, supports itself in part through several community-operated businesses and many of the problems, including the expression of symptoms that in other places would be resolved by professionals, are handled by the community. ${ }^{12,13}$

\section{Conclusion}

Helping people with mental illness achieve economically sustainable community lives will require us to alter current beliefs and practices. One road to broad community participation may run through a more fully developed identity community rather than through mental health service providers. Many people with psychiatric disorders may opt to have successful community lives by remaining principally within those identity communities or by moving between their identity community and the broad community. Many core supports can be provided through the social lives and infrastructure of the identity community rather than through services. Such evolving communities would need community organisers rather than mental health service providers. When mental health services are needed, they should not dominate the life of service recipients but rather occupy the same limited role that other health services have in people's lives; and public funding of services should not be used for supports that can be achieved through economically sustainable community and market approaches.

The identity community model is consistent with current ideas about recovery. Understood correctly, recovery is a process, not an outcome. People in recovery need something other than services to facilitate that process. Identity community development provides one such approach.

\section{About the authors}

James M. Mandiberg PhD, is an Assistant Professor of Management and Social Enterprise at Columbia University School of Social Work, Columbia University, New York, USA; Richard Warner MB DPM, is Director, Colorado Recovery, Boulder, Colorado, and Professor of Psychiatry at the University of Colorado, Denver, Colorado, USA.

\section{References}

1 Warner R. Local projects of the World Psychiatric Association programme to reduce stigma and discrimination. Psychiatr Serv 2005. 56: $570-5$.

2 Evans-Lacko S, London J, Little K, Henderson C, Thornicroft G. Evaluation of a brief anti-stigma campaign in Cambridge: do shortterm campaigns work? BMC Public Health 2010; 10: 339.

3 Mandiberg JM, Edwards M. Collective identity formation in the Menta Health Clubhouse Community. Int J Self-Help Self-Care 2013; 7: 19-39.

4 Mandiberg JM. Another way: enclave communities for people with mental illness. Am J Orthopsychiatry 2010; 80: 170-6.

5 Berry JW. Acculturation: living successfully in two cultures. Int J Intercult Relations 2005; 29: 697-712.

6 Mandiberg JM, Warner R. Business development and marketing within communities of social service clients. J Bus Res 2012; 65: 1736-42.

7 Mandiberg JM. Commentary: the failure of social inclusion: an alternative approach through community development. Psychiatr Serv 2012; 63: 458 .

8 Mandiberg JM, Telles L. The Santa Clara County Clustered Apartment Project. Psychosoc Rehabil J 1990; 14: 21-8.

9 Mandiberg JM. Can interdependent mutual support function as an alternative to hospitalization? In Alternatives to Hospitalization for Acute Psychiatric Care (ed. R Warner): 193-210. American Psychiatric Press, 1995.

10 Leff JP, Warner R. Social Inclusion of People with Mental Illness. Cambridge University Press, 2006.

11 Warner R, Mandiberg J. An update on affirmative businesses or social firms for people with mental illness. Psychiatr Serv 2006; 57: 1488-92.

12 Kimura M, Mukaiyachi I, Ito E. The House of Bethel and consumer-run businesses: an innovative approach to psychiatric rehabilitation. Can J Commun Ment Health 2002; 21: 69-77.

13 Nakamura K. Bethel: Community and Schizophrenia in Northern Japan (DVD). Manic Productions, 2010 